\title{
Representations of Generalized Inverses and Drazin Inverse of Partitioned Matrix with Banachiewicz-Schur Forms
}

\author{
Xiaoji Liu, ${ }^{1}$ Hongwei Jin, ${ }^{1}$ and Jelena Višnjićc ${ }^{2}$ \\ ${ }^{1}$ Faculty of Science, Guangxi University for Nationalities, Nanning 530006, China
}

${ }^{2}$ Faculty of Medicine, University of Niš, 18000 Niš, Serbia

Correspondence should be addressed to Xiaoji Liu; xiaojiliu72@126.com

Received 15 July 2016; Accepted 6 September 2016

Academic Editor: Gen Qi Xu

Copyright (C) 2016 Xiaoji Liu et al. This is an open access article distributed under the Creative Commons Attribution License, which permits unrestricted use, distribution, and reproduction in any medium, provided the original work is properly cited.

Representations of $\{1,2,3\}$-inverses, $\{1,2,4\}$-inverses, and Drazin inverse of a partitioned matrix $M=\left[\begin{array}{ll}A & B \\ C & D\end{array}\right]$ related to the generalized Schur complement are studied. First, we give the necessary and sufficient conditions under which $\{1,2,3\}$-inverses, $\{1,2,4\}$-inverses, and group inverse of a $2 \times 2$ block matrix can be represented in the Banachiewicz-Schur forms. Some results from the paper of Cvetković-Ilić, 2009, are generalized. Also, we expressed the quotient property and the first Sylvester identity in terms of the generalized Schur complement.

\section{Introduction}

Let $\mathbb{C}^{m \times n}$ denote the set of all $m \times n$ complex matrices. We use $R(A), N(A)$, and $r(A)$ to denote the range, the null space, and the rank of a matrix $A$, respectively. The smallest nonnegative integer $k$ such that $r\left(A^{k+1}\right)=r\left(A^{k}\right)$ is the index of $A$ which is denoted by ind $(A)$. If ind $(A)=r$, the Drazin inverse of $A$ is the unique matrix $X \in \mathbb{C}^{m \times m}$ satisfying

$$
\begin{aligned}
X A X & =X, \\
A X & =X A, \\
A^{k+1} X & =A^{k},
\end{aligned}
$$

for all $k \geq r$. The Drazin inverse of $A$ is denoted by $A^{D}$. In particular, when ind $(A)=1$, the matrix $A^{D}$ is called the group inverse of $A$ and it is denoted by $A^{\#}$.

Recall that the Moore-Penrose inverse of a matrix $A \in$ $\mathbb{C}^{m \times n}$ is a matrix $X \in \mathbb{C}^{n \times m}$ which satisfies
(1) $A X A=A$,
(2) $X A X=X$,
(3) $(A X)^{*}=A X$,
(4) $(X A)^{*}=X A$.

The Moore-Penrose inverse of $A$ is unique and it is denoted by $A^{\dagger}$.

For any $A \in \mathbb{C}^{m \times n}$, let $A\{i, j, \ldots, k\}$ denote the set of all $X \in \mathbb{C}^{n \times m}$ which satisfy equations $(i),(j), \ldots,(k)$ of $(2)$. In this case $X$ is a $\{i, j, \ldots, k\}$-inverse of $A$, which we denote by $A^{(i, j, \ldots, k)}$. Evidently, $A\{1,2,3,4\}=\left\{A^{\dagger}\right\}$.

For a complex matrix $M$ of the form

$$
M=\left[\begin{array}{ll}
A & B \\
C & D
\end{array}\right] \in \mathbb{C}^{(m+p) \times(n+p)},
$$

in the case when $A$ is invertible, the Schur complement of $A$ in $M$ is defined by $S=D-C A^{-1} B$. Similarly, if $D$ is invertible, then the Schur complement of $D$ in $M$ is defined by $G=A-$ $B D^{-1} C$.

It is well-known that if $A \in \mathbb{C}^{m \times m}$ is nonsingular then the invertibility of a matrix $M$ is equivalent to the invertibility of the Schur complement of $A$ in $M$ and in that case the inverse of $M$ is given by

$$
M^{-1}=\left[\begin{array}{cc}
A^{-1}+A^{-1} B S^{-1} C A^{-1} & -A^{-1} B S^{-1} \\
-S^{-1} C A^{-1} & S^{-1}
\end{array}\right] .
$$

Expression (4) is called the Banachiewicz-Schur form of the matrix $M$. The notation of Schur complement was 
first introduced by Schur [1]. G. E. Trapp first defined the generalized Schur complement where the ordinary inverse was replaced by the generalized inverse. For a matrix $M \in$ $\mathbb{C}^{(m+p) \times(n+p)}$ given by (3) and any fixed generalized inverse $A^{-} \in A\{1\}$, the generalized Schur complement of $A$ in $M$ is defined by

$$
S\left(A^{-}\right)=D-C A^{-} B .
$$

Similarly, for some fixed $D^{-} \in D\{1\}$, the generalized Schur complement of $D$ in $M$ is defined by

$$
G\left(D^{-}\right)=A-B D^{-} C
$$

The Schur complements and generalized Schur complements were studied by a number of authors, which have applications in statistics, matrix theory, electrical network theory, discrete-time regulator problem, sophisticated techniques, and some other fields. It has been evident that the Schur complement plays an important role in many aspects of a matrix theory (see [2-8]).

Let

$$
X=\left[\begin{array}{cc}
A^{-}+A^{-} B S^{-} C A^{-} & -A^{-} B S^{-} \\
-S^{-} C A^{-} & S^{-}
\end{array}\right],
$$

where $A^{-} \in A\{1\}, S=D-C A^{-} B$, and $S^{-} \in S\{1\}$, and let

$$
Y=\left[\begin{array}{cc}
G^{-} & -G^{-} B D^{-} \\
-D^{-} C G^{-} & D^{-}+D^{-} C G^{-} B D^{-}
\end{array}\right],
$$

where $D^{-} \in D\{1\}, G=A-B D^{-} C$, and $G^{-} \in G\{1\}$.

For convenience, we will firstly state the following notations which are helpful in the proofs. We denote

$$
\begin{aligned}
& E_{\alpha}=I-\alpha^{-} \alpha, \\
& F_{\alpha}=I-\alpha \alpha^{-}, \\
& \alpha^{\pi}=I-\alpha \alpha^{D},
\end{aligned}
$$

$$
\alpha \in\{A, S, D, G\} .
$$

In this paper, we will establish necessary and sufficient conditions under which matrices $X$ and $Y$ given by (7) and (8), respectively, belong to a given class of generalized inverses of the matrix $M$ given by (3). Our interest focuses on the cases when $\{1,2,3\}$-inverses, $\{1,2,4\}$-inverses, and group inverse of a $2 \times 2$ block matrix $M$ are given by the Banachiewicz-Schur form. Also, we give representations of the Drazin inverse of $2 \times 2$ block matrix $M$ under some conditions relating to the Schur complement of $M$, which generalized the results studied by Cvetković-Ilić [9]. Also, the quotient property and the first Sylvester identity based on the MP-inverse, group inverse, and Drazin inverse are given.

We need some auxiliary lemmas.

Lemma 1 (see [10]). Let $M, S$, and $X$ be defined by (3), (5), and (7), respectively. Then $X \in M\{1,3\}$ if and only if

$$
\begin{aligned}
& A^{-} \in A\{1,3\}, \\
& S^{-} \in S\{1,3\},
\end{aligned}
$$

$$
\begin{aligned}
& F_{A} B=0, \\
& F_{S} C=0,
\end{aligned}
$$

the last two conditions being independent of the choice of $A^{-} \epsilon$ $A\{1\}$ and $S^{-} \in S\{1\}$ involved in $F_{A}$ and $F_{S}$.

Lemma 2 (see [10]). Let $M, S$, and $X$ be defined by (3), (5), and (7), respectively. Then $X \in M\{1,4\}$ if and only if

$$
\begin{aligned}
A^{-} & \in A\{1,4\}, \\
S^{-} & \in S\{1,4\}, \\
B E_{S} & =0, \\
C E_{A} & =0,
\end{aligned}
$$

the last two conditions being independent of the choice of $A^{-} \epsilon$ $A\{1\}$ and $S^{-} \in S\{1\}$ involved in $F_{A}$ and $F_{S}$.

Lemma 3 (see [10]). Let $M, S$, and $X$ be defined by (3), (5), and (7), respectively. Then $X \in M\{2\}$ if and only if

$$
\begin{aligned}
& A^{-} \in A\{1,2\}, \\
& S^{-} \in S\{2\} .
\end{aligned}
$$

Lemma 4. Let $M, S$, and $X$ be defined by (3), (5), and (7), respectively. Then $X \in M\{1,2,3\}$ if and only if

$$
\begin{aligned}
A^{-} & \in A\{1,2,3\}, \\
S^{-} & \in S\{1,2,3\}, \\
F_{A} B & =0, \\
F_{S} C & =0,
\end{aligned}
$$

the last two conditions being independent of the choice of $A^{-} \epsilon$ $A\{1\}$ and $S^{-} \in S\{1\}$ involved in $F_{A}$ and $F_{S}$.

Proof. The conclusion follows by Lemmas 1 and 2 .

Lemma 5. Let $M, S$, and $X$ be defined by (3), (5), and (7), respectively. Then $X \in M\{1,2,4\}$ if and only if

$$
\begin{aligned}
A^{-} & \in A\{1,2,4\}, \\
S^{-} & \in S\{1,2,4\}, \\
B E_{S} & =0, \\
C E_{A} & =0,
\end{aligned}
$$

the last two conditions being independent of the choice of $A^{-} \epsilon$ $A\{1\}$ and $S^{-} \in S\{1\}$ involved in $E_{A}$ and $E_{S}$.

Using the same method as in [10], we can prove the following. 
Lemma 6. Let $M, S$, and $X$ be defined by (3), (5), and (7), respectively. Then $Y \in M\{1,2,3\}$ if and only if

$$
\begin{aligned}
D^{-} & \in D\{1,2,3\}, \\
G^{-} & \in G\{1,2,3\}, \\
F_{G} B & =0, \\
F_{D} C & =0,
\end{aligned}
$$

the last two conditions being independent of the choice of $D^{-} \epsilon$ $D\{1\}$ and $G^{-} \in G\{1\}$ involved in $F_{D}$ and $F_{G}$.

Lemma 7. Let $M, S$, and $X$ be defined by (3), (5), and (7), respectively. Then $Y \in M\{1,2,4\}$ if and only if

$$
\begin{aligned}
D^{-} & \in D\{1,2,4\}, \\
G^{-} & \in G\{1,2,4\}, \\
B E_{D} & =0, \\
C E_{G} & =0,
\end{aligned}
$$

the last two conditions being independent of the choice of $D^{-} \in$ $D\{1\}$ and $G^{-} \in G\{1\}$ involved in $F_{D}$ and $F_{G}$.

Lemma 8 (see [11]). Let $M, S$, and $X$ be defined by (3), (5), and (7), respectively. Then $X=M^{\#}$ if and only if

$$
\begin{aligned}
A^{-} & =A^{\#}, \\
S^{-} & =S^{\#}, \\
F_{A} B & =0, \\
F_{S} C & =0, \\
C E_{A} & =0, \\
B E_{S} & =0 .
\end{aligned}
$$

\section{Representations of $\{1,2,3\}$-Inverse, $\{1,2,4\}$ - Inverse, and Group Inverse in terms of Banachiewicz-Schur Forms}

Baksalary and Styan [10] presented the necessary and sufficient conditions under which $\{1\},\{2\},\{1,2\},\{1,3\}$, and $\{1,4\}$-inverses of block matrix $M$ can be represented by the Banachiewicz-Schur form.

Sheng and Chen [12] considered sufficient conditions under which $M^{\dagger}, M^{D}$, and $M^{\#}$ can be represented by both of the Banachiewicz-Schur forms at the same time.

Cvetković-Ilić [9] gave necessary and sufficient conditions which ensure the representation of the MP-inverse of a block matrix $M$ by both of the Banachiewicz-Schur forms.

In this section, we will present necessary and sufficient conditions under which $\{1,2,3\},\{1,2,4\}$-inverses and the group inverse of a partitioned matrix $M$ can be represented by the Banachiewicz-Schur form.
For convenience, we first introduce the following notations:

$$
\begin{aligned}
& N_{1}\{i, j, k\}=\{X \\
& =\left[\begin{array}{cc}
A^{-}+A^{-} B S^{-} C A^{-} & -A^{-} B S^{-} \\
-S^{-} C A^{-} & S^{-}
\end{array}\right]: A^{-} \\
& \left.\quad \in A\{i, j, k\}, S=D-C A^{-} B, S^{-} \in S\{i, j, k\}\right\}, \\
& N_{2}\{i, j, k\}=\left\{Y \quad-G^{-} B D^{-}\right. \\
& =\left[\begin{array}{c}
G \\
-D^{-} C G^{-} \quad D^{-}+D^{-} C G^{-} B D^{-}
\end{array}\right]: D^{-} \\
& \left.\in D\{i, j, k\}, G=A-B D^{-} C, G^{-} \in G\{i, j, k\}\right\} .
\end{aligned}
$$

Theorem 9. Let $M, S$, and $G$ be defined by (3), (5), and (6), respectively. Then the following happens.

(i) $N_{1}\{1,2,3\} \subseteq N_{2}\{1,2,3\} \subseteq M\{1,2,3\}$ if and only if for arbitrary $A^{-} \in A\{1,2,3\}, D^{-} \in D\{1,2,3\}$ and $G^{-} \in G\{1,2,3\}$,

$$
\begin{aligned}
& F_{A} B=0, \\
& F_{D} C=0, \\
& F_{G} B=0,
\end{aligned}
$$

and for any $A^{-} \in A\{1,2,3\}$ and any $S^{-} \in S\{1,2,3\}$, there exists $D^{-} \in D\{1,2,3\}$ such that

$$
\begin{aligned}
F_{D} & =F_{S}, \\
E_{D} S^{-} & =0, \\
E_{S} D^{-} & =0 .
\end{aligned}
$$

(ii) $N_{2}\{1,2,3\} \subseteq N_{1}\{1,2,3\} \subseteq M\{1,2,3\}$ if and only if for arbitrary $A^{-} \in A\{1,2,3\}, D^{-} \in D\{1,2,3\}$ and $S^{-} \in S\{1,2,3\}$,

$$
\begin{aligned}
& F_{A} B=0, \\
& F_{D} C=0, \\
& F_{S} C=0,
\end{aligned}
$$

and for any $D^{-} \in D\{1,2,3\}$ and any $G^{-} \in G\{1,2,3\}$, there exists $A^{-} \in A\{1,2,3\}$ such that

$$
\begin{aligned}
F_{A} & =F_{G}, \\
E_{A} G^{-} & =0, \\
E_{G} A^{-} & =0 .
\end{aligned}
$$

(iii) $N_{1}\{1,2,3\}=N_{2}\{1,2,3\} \subseteq M\{1,2,3\}$ if and only if for arbitrary $A^{-} \in A\{1,2,3\}, D^{-} \in D\{1,2,3\}$,

$$
\begin{aligned}
& F_{A} B=0, \\
& F_{D} C=0,
\end{aligned}
$$


and for any $A^{-} \in A\{1,2,3\}$ and any $S^{-} \in S\{1,2,3\}$, there exists $D^{-} \in D\{1,2,3\}$ such that $F_{D}=F_{S}, E_{D} S^{-}=0$, and $E_{S} D^{-}=0$, and for any $D^{-} \in D\{1,2,3\}$ and $G^{-} \in G\{1,2,3\}$, there exists $A^{-} \in A\{1,2,3\}$ such that $F_{A}=F_{G}, E_{A} G^{-}=0$, and $E_{G} A^{-}=0$.

Proof. (i) ( $\Leftarrow$ :) By Lemma 6 , we have that arbitrary $Y \in$ $N_{2}\{1,2,3\}$ belongs to $M\{1,2,3\}$. Now, we only need to prove that $N_{1}\{1,2,3\} \subseteq N_{2}\{1,2,3\}$. Take arbitrary

$$
X_{1}=\left[\begin{array}{cc}
A_{1}^{-}+A_{1}^{-} B S_{1}^{-} C A_{1}^{-} & -A_{1}^{-} B S_{1}^{-} \\
-S_{1}^{-} C A_{1}^{-} & S_{1}^{-}
\end{array}\right] \in N_{1}\{1,2,3\} .
$$

By assumption, for $S_{1}^{-}$, there exists $\{1,2,3\}$-inverse of $D$ which we denote by $D_{1}^{-}$such that $F_{D}=F_{S_{1}}, E_{D_{1}} S_{1}^{-}=0$, and $E_{S_{1}} D_{1}^{-}=0$. Let $G=A-B D_{1}^{-} C$. We will prove that $G_{1}^{-}=$ $A_{1}^{-}+A_{1}^{-} B S_{1}^{-} C A_{1}^{-} \in G\{1,2,3\}$. By computation, we get

$$
\begin{aligned}
G G_{1}^{-}= & \left(A-B D_{1}^{-} C\right)\left(A_{1}^{-}+A_{1}^{-} B S_{1}^{-} C A_{1}^{-}\right) \\
= & A A_{1}^{-}+A A_{1}^{-} B S_{1}^{-} C A_{1}^{-}-B D_{1}^{-} C A_{1}^{-} \\
& -B D_{1}^{-} C A_{1}^{-} B S_{1}^{-} C A_{1}^{-} \\
= & A A_{1}^{-}+B S_{1}^{-} C A_{1}^{-}-B D_{1}^{-} C A_{1}^{-}-B D_{1}^{-} D S_{1}^{-} C A_{1}^{-} \\
& +B D_{1}^{-} S_{1} S_{1}^{-} C A_{1}^{-}=A A_{1}^{-},
\end{aligned}
$$

which implies $G G_{1}^{-} G=A A_{1}^{-}\left(A-B D_{1}^{-} C\right)=A-B D_{1}^{-} C=G$. Also,

$$
\begin{aligned}
G_{1}^{-} G G_{1}^{-} & =\left(A_{1}^{-}+A_{1}^{-} B S_{1}^{-} C A_{1}^{-}\right) A A_{1}^{-} \\
& =A_{1}^{-}+A_{1}^{-} B S_{1}^{-} C A_{1}^{-}=G_{1}^{-},
\end{aligned}
$$

so, $G_{1}^{-} \in G\{1,2,3\}$. Since $F_{D}=F_{S_{1}}, E_{D} S_{1}^{-}=0$, and $E_{S_{1}} D_{1}^{-}=0$, a simple computation shows that

$$
\begin{aligned}
D_{1}^{-} C G_{1}^{-}= & D_{1}^{-} C\left(A_{1}^{-}+A_{1}^{-} B S_{1}^{-} C A_{1}^{-}\right) \\
= & D_{1}^{-} C A_{1}^{-}+D_{1}^{-} C A_{1}^{-} B S_{1}^{-} C A_{1}^{-} \\
= & D_{1}^{-} C A_{1}^{-}+D_{1}^{-}\left(D-S_{1}\right) S_{1}^{-} C A_{1}^{-} \\
= & D_{1}^{-} C A_{1}^{-}+D_{1}^{-} D S_{1}^{-} C A_{1}^{-} \\
& -D_{1}^{-} S_{1} S_{1}^{-} C A_{1}^{-}=S_{1}^{-} C A_{1}^{-}, \\
G_{1}^{-} B D_{1}^{-}= & \left(A_{1}^{-}+A_{1}^{-} B S_{1}^{-} C A_{1}^{-}\right) B D_{1}^{-} \\
= & A_{1}^{-} B D_{1}^{-}+A_{1}^{-} B S_{1}^{-} C A_{1}^{-} B D_{1}^{-} \\
= & A_{1}^{-} B D_{1}^{-}+A_{1}^{-} B S_{1}^{-}\left(D-S_{1}\right) D_{1}^{-} \\
= & A_{1}^{-} B D_{1}^{-}+A_{1}^{-} B S_{1}^{-} D D_{1}^{-} \\
& -A_{1}^{-} B S_{1}^{-} S_{1} D_{1}^{-}=A_{1}^{-} B S_{1}^{-},
\end{aligned}
$$

$$
\begin{aligned}
D_{1}^{-}+D_{1}^{-} C G_{1}^{-} B D_{1}^{-}= & D_{1}^{-} \\
& +D_{1}^{-} C\left(A_{1}^{-}+A_{1}^{-} B S_{1}^{-} C A_{1}^{-}\right) B D_{1}^{-} \\
= & D_{1}^{-}+D_{1}^{-} C A_{1}^{-} B D_{1}^{-} \\
& +D_{1}^{-} C A_{1}^{-} B S_{1}^{-} C A_{1}^{-} B D_{1}^{-} \\
= & D_{1}^{-}+D_{1}^{-}\left(D-S_{1}\right) D_{1}^{-} \\
& +D_{1}^{-}\left(D-S_{1}\right) S_{1}^{-}\left(D-S_{1}\right) D_{1}^{-} \\
= & S_{1}^{-} .
\end{aligned}
$$

Thus,

$$
\begin{aligned}
X_{1} & =\left[\begin{array}{cc}
A_{1}^{-}+A_{1}^{-} B S_{1}^{-} C A_{1}^{-} & -A_{1}^{-} B S_{1}^{-} \\
-S_{1}^{-} C A_{1}^{-} & S_{1}^{-}
\end{array}\right] \\
& =\left[\begin{array}{cc}
G_{1}^{-} & -G_{1}^{-} B D_{1}^{-} \\
-D_{1}^{-} C G_{1}^{-} & D_{1}^{-}+D_{1}^{-} C G_{1}^{-} B D_{1}^{-}
\end{array}\right] \in N_{2} .
\end{aligned}
$$

Therefore, $N_{1} \subseteq N_{2} \subseteq M\{1,2,3\}$.

$\left(\Rightarrow\right.$ :) Since $N_{1}\{1,2,3\} \subseteq M\{1,2,3\}$ and $N_{2}\{1,2,3\} \subseteq$ $M\{1,2,3\}$, by Lemmas 4 and 6 , we get that, for arbitrary $A^{-} \in A\{1,2,3\}, D^{-} \in D\{1,2,3\}$, and $G^{-} \in G\{1,2,3\}$, the following hold: $F_{A} B=0, F_{G} B=0$, and $F_{D} C=0$. Moreover, by $N_{1}\{1,2,3\} \subseteq N_{2}\{1,2,3\}$, it follows that, for any $X \in N_{1}\{1,2,3\}$, there exists some $Y \in N_{2}\{1,2,3\}$ such that $X=Y$. Hence, for any $A^{-} \in A\{1,2,3\}, S^{-} \in S\{1,2,3\}$, there exist $D^{-} \in D\{1,2,3\}$ and $G^{-} \in G\{1,2,3\}$ such that

$$
\begin{gathered}
{\left[\begin{array}{cc}
A^{-}+A^{-} B S^{-} C A^{-} & -A^{-} B S^{-} \\
-S^{-} C A^{-} & S^{-}
\end{array}\right]} \\
\quad=\left[\begin{array}{cc}
G^{-} & -G^{-} B D^{-} \\
-D^{-} C G^{-} & D^{-}+D^{-} C G^{-} B D^{-}
\end{array}\right] .
\end{gathered}
$$

A simple computation shows that

$$
\begin{aligned}
S S^{-}= & \left(D-C A^{-} B\right)\left(D^{-}+D^{-} C G^{-} B D^{-}\right) \\
= & D D^{-}+D D^{-} C G^{-} B D^{-}-C A^{-} B D^{-} \\
& -C A^{-} A G^{-} B D^{-}+C A^{-} G G^{-} B D^{-} \\
= & D D^{-}+C\left(I-A^{-} A\right) G^{-} B D^{-} \\
= & D D^{-}+C\left(I-A^{-} A\right) A^{-} B S^{-}=D D^{-} ;
\end{aligned}
$$

thus, we get $F_{S}=F_{D}$. Since

$$
\begin{aligned}
D^{-}+D^{-} C G^{-} B D^{-} & =D^{-}+S^{-} C A^{-} B D^{-} \\
& =D^{-}+S^{-}(D-S) D^{-} \\
& =D^{-}+S^{-} D D^{-}-S^{-} S D^{-}=S^{-},
\end{aligned}
$$


we get $D^{-}=S^{-} S D^{-}$; that is, $E_{S} D^{-}=0$. Similarly,

$$
\begin{aligned}
D^{-}+D^{-} C G^{-} B D^{-} & =D^{-}+D^{-} C A^{-} B S^{-} \\
& =D^{-}+D^{-}(D-S) S^{-} \\
& =D^{-}+D^{-} D S^{-}-D^{-} S S^{-}=S^{-},
\end{aligned}
$$

which implies $E_{D} S^{-}=0$.

(ii) $\left(\Leftarrow\right.$ :) Similar to $(i)$, since $F_{A} B=0$ and $F_{S} C=0$, we have $N_{1}\{1,2,3\} \subseteq M\{1,2,3\}$. Now, we will prove that $N_{2}\{1,2,3\} \subseteq N_{1}\{1,2,3\}$. Take arbitrary

$$
Y_{1}=\left[\begin{array}{cc}
G_{1}^{-} & -G_{1}^{-} B D_{1}^{-} \\
-D_{1}^{-} C G_{1}^{-} & D_{1}^{-}+D_{1}^{-} C G_{1}^{-} B D_{1}^{-}
\end{array}\right] \in N_{2}\{1,2,3\}
$$

For $G_{1}^{-}$, there exists $\{1,2,3\}$-inverse of $A$ which we denote by $A_{1}^{-}$such that $F_{A}=F_{G_{1}}, E_{A} G_{1}^{-}=0$, and $E_{G_{1}} A_{1}^{-}=0$. Let $S=$ $D-C A_{1}^{-} B$. A simple computation shows that

$$
\begin{aligned}
S_{1}^{-} & =D_{1}^{-}+D_{1}^{-} C G_{1}^{-} B D_{1}^{-} \in S\{1,2,3\}, \\
S_{1}^{-} C A_{1}^{-} & =D_{1}^{-} C G_{1}^{-}, \\
A_{1}^{-} B S_{1}^{-} & =G_{1}^{-} B D_{1}^{-}, \\
A_{1}^{-}+A_{1}^{-} B S_{1}^{-} C A_{1}^{-} & =G_{1}^{-} .
\end{aligned}
$$

Hence,

$$
\begin{aligned}
Y_{1} & =\left[\begin{array}{cc}
G_{1}^{-} & -G_{1}^{-} B D_{1}^{-} \\
-D_{1}^{-} C G_{1}^{-} & D_{1}^{-}+D_{1}^{-} C G_{1}^{-} B D_{1}^{-}
\end{array}\right] \\
& =\left[\begin{array}{cc}
A_{1}^{-}+A_{1}^{-} B S_{1}^{-} C A_{1}^{-} & -A_{1}^{-} B S_{1}^{-} \\
-S_{1}^{-} C A_{1}^{-} & S_{1}^{-}
\end{array}\right] \in N_{1}\{1,2,3\} .
\end{aligned}
$$

Therefore, $N_{2}\{1,2,3\} \subseteq N_{1}\{1,2,3\} \subseteq M\{1,2,3\}$. The reverse part of the proof is similar to (i).

(iii) Combining (i) and (ii), we get (iii).

The case for $\{1,2,4\}$-inverses is treated completely analogously and the corresponding result follows by taking adjoint.

Theorem 10. Let $M, S$, and $G$ be defined by (3), (5), and (6), respectively. Then the following happens.

(i) $N_{1}\{1,2,4\} \subseteq N_{2}\{1,2,4\} \subseteq M\{1,2,4\}$ if and only if for arbitrary $A^{-} \in A\{1,2,4\}, D^{-} \in D\{1,2,4\}$, and $G^{-} \in$ $G\{1,2,4\}$,

$$
\begin{aligned}
& C E_{A}=0, \\
& B E_{D}=0, \\
& C E_{G}=0,
\end{aligned}
$$

and for any $A^{-} \in A\{1,2,4\}$ and any $S^{-} \in S\{1,2,4\}$, there exists $D^{-} \in D\{1,2,4\}$ such that

$$
\begin{aligned}
E_{S} & =E_{D}, \\
S^{-} F_{D} & =0, \\
D^{-} F_{S} & =0 .
\end{aligned}
$$

(ii) $N_{2}\{1,2,4\} \subseteq N_{1}\{1,2,4\} \subseteq M\{1,2,4\}$ if and only if for arbitrary $A^{-} \in A\{1,2,4\}, D^{-} \in D\{1,2,4\}$, and $S^{-} \in S\{1,2,4\}$,

$$
\begin{aligned}
& C E_{A}=0, \\
& B E_{D}=0, \\
& B E_{S}=0,
\end{aligned}
$$

and for any $D^{-} \in D\{1,2,4\}$ and any $G^{-} \in G\{1,2,4\}$, there exists $A^{-} \in A\{1,2,4\}$ such that

$$
\begin{aligned}
E_{A} & =E_{G}, \\
G^{-} F_{A} & =0, \\
A^{-} F_{G} & =0 .
\end{aligned}
$$

(iii) $N_{1}\{1,2,4\}=N_{2}\{1,2,4\} \subseteq M\{1,2,4\}$ if and only iffor arbitrary $A^{-} \in A\{1,2,4\}, D^{-} \in D\{1,2,4\}$,

$$
\begin{aligned}
& C E_{A}=0, \\
& B E_{D}=0,
\end{aligned}
$$

and for any $A^{-} \in A\{1,2,4\}$ and any $S^{-} \in S\{1,2,4\}$, there exists $D^{-} \in D\{1,2,4\}$ such that $E_{S}=E_{D}, S^{-} F_{D}=0$, and $D^{-} F_{S}=0$, and for any $D^{-} \in D\{1,2,4\}$ and any $G^{-} \in G\{1,2,4\}$, there exists $A^{-} \in A\{1,2,4\}$ such that $E_{A}=E_{G}, G^{-} F_{A}=0$, and $A^{-} F_{G}=0$.

Similarly, we get the following results.

Theorem 11. Let $M, S$, and $G$ be defined by (3), (5), and (6), respectively. Then the following happens.

(i) For arbitrary $X \in N_{1}\{1,2,3\}$ given by (7), there exists $Y \in N_{2}\{1,2,3\}$ such that $X=Y \in M\{1,2,3\}$ if and only if $A^{-} \in A\{1,2,3\}$ and $S^{-} \in S\{1,2,3\}$ and there exist $D^{-} \in$ $D\{1,2,3\}$ and $G^{-} \in G\{1,2,3\}$ such that

$$
\begin{aligned}
F_{A} B & =0, \\
F_{D} C & =0, \\
F_{G} B & =0, \\
F_{D} & =F_{S}, \\
E_{D} S^{-} & =0, \\
E_{S} D^{-} & =0 .
\end{aligned}
$$

(ii) For arbitrary $Y \in N_{2}\{1,2,3\}$ given by (8), there exists $X \in N_{1}\{1,2,3\}$ such that $X=Y \in M\{1,2,3\}$ if and only if $D^{-} \in D\{1,2,3\}$ and $G^{-} \in G\{1,2,3\}$ and there exist $A^{-} \in$ $A\{1,2,3\}$ and $S^{-} \in S\{1,2,3\}$ such that

$$
\begin{aligned}
& F_{A} B=0, \\
& F_{D} C=0, \\
& F_{S} C=0,
\end{aligned}
$$




$$
\begin{aligned}
F_{A} & =F_{G}, \\
E_{A} G^{-} & =0, \\
E_{G} A^{-} & =0 .
\end{aligned}
$$

Theorem 12. Let $M, S$, and $G$ be defined by (3), (5), and (6), respectively. Then the following happens.

(i) For arbitrary $X \in N_{1}\{1,2,4\}$ given by (7), there exists $Y \in N_{2}\{1,2,4\}$ such that $X=Y \in M\{1,2,4\}$ if and only if $A^{-} \in A\{1,2,4\}$ and $S^{-} \in S\{1,2,4\}$ and there exist $D^{-} \in$ $D\{1,2,4\}$ and $G^{-} \in G\{1,2,4\}$ such that

$$
\begin{aligned}
C E_{A} & =0, \\
B E_{D} & =0, \\
C E_{G} & =0, \\
E_{S} & =E_{D}, \\
S^{-} F_{D} & =0, \\
D^{-} F_{S} & =0 .
\end{aligned}
$$

(ii) For arbitrary $Y \in N_{2}\{1,2,4\}$ given by (8), there exists $X \in N_{1}\{1,2,4\}$ such that $X=Y \in M\{1,2,4\}$ if and only if $D^{-} \in D\{1,2,4\}$ and $G^{-} \in G\{1,2,4\}$ and there exist $A^{-} \in$ $A\{1,2,4\}$ and $S^{-} \in S\{1,2,4\}$ such that

$$
\begin{aligned}
C E_{A} & =0, \\
B E_{D} & =0, \\
B E_{S} & =0, \\
E_{A} & =E_{G}, \\
G^{-} F_{A} & =0, \\
A^{-} F_{G} & =0 .
\end{aligned}
$$

Theorem 13. Let $M, S$, and $G$ be defined by (3), (5), and (6), respectively. Then

$$
\begin{aligned}
M^{\dagger} & =\left[\begin{array}{cc}
A^{-}+A^{-} B S^{-} C A^{-} & -A^{-} B S^{-} \\
-S^{-} C A^{-} & S^{-}
\end{array}\right] \\
& =\left[\begin{array}{cc}
G^{-} & -G^{-} B D^{-} \\
-D^{-} C G^{-} & D^{-}+D^{-} C G^{-} B D^{-}
\end{array}\right]
\end{aligned}
$$

if and only if

$$
\begin{aligned}
A^{-} & =A^{\dagger}, \\
D^{-} & =D^{\dagger}, \\
S^{-} & =S^{\dagger}, \\
G^{-} & =G^{\dagger}, \\
F_{A} B & =0,
\end{aligned}
$$

$$
\begin{aligned}
F_{D} C & =0, \\
C E_{A} & =0, \\
B E_{D} & =0, \\
E_{D} & =E_{S}, \\
F_{D} & =F_{S} .
\end{aligned}
$$

Proof. By Theorems 11 and 12, we get

$$
\begin{aligned}
M^{\dagger} & =\left[\begin{array}{cc}
A^{-}+A^{-} B S^{-} C A^{-} & -A^{-} B S^{-} \\
-S^{-} C A^{-} & S^{-}
\end{array}\right] \\
& =\left[\begin{array}{cc}
G^{-} & -G^{-} B D^{-} \\
-D^{-} C G^{-} & D^{-}+D^{-} C G^{-} B D^{-}
\end{array}\right]
\end{aligned}
$$

if and only if

$$
\begin{aligned}
A^{-} & =A^{\dagger}, \\
D^{-} & =D^{\dagger}, \\
S^{-} & =S^{\dagger}, \\
G^{-} & =G^{\dagger}, \\
E_{D} S^{-} & =0, \\
E_{S} D^{-} & =0, \\
E_{A} G^{-} & =0, \\
E_{G} A^{-} & =0, \\
D^{-} F_{S} & =0, \\
S^{-} F_{D} & =0, \\
G^{-} F_{A} & =0, \\
A^{-} F_{G} & =0, \\
F_{A} B & =0, \\
F_{D} C & =0, \\
C E_{A} & =0, \\
B E_{D} & =0, \\
E_{S} & =E_{D}, \\
E_{A} & =E_{G}, \\
F_{D} & =F_{S}, \\
F_{A} & =F_{G},
\end{aligned}
$$


Since $E_{S}=E_{D}$ and $E_{D} D^{-}=0$, then $E_{S} D^{-}=0$. Thus,

$$
\begin{aligned}
& E_{S}=E_{D}, \\
& E_{A}=E_{G}, \\
& F_{D}=F_{S}, \\
& F_{A}=F_{G}
\end{aligned}
$$

implies

$$
\begin{aligned}
& E_{D} S^{-}=0, \\
& E_{S} D^{-}=0, \\
& E_{A} G^{-}=0, \\
& E_{G} A^{-}=0, \\
& D^{-} F_{S}=0, \\
& S^{-} F_{D}=0, \\
& G^{-} F_{A}=0, \\
& A^{-} F_{G}=0 .
\end{aligned}
$$

Now, we only need to prove that

$$
\begin{aligned}
F_{A} B & =0, \\
F_{D} C & =0, \\
C E_{A} & =0, \\
B E_{D} & =0, \\
E_{S} & =E_{D}, \\
E_{A} & =E_{G}, \\
F_{D} & =F_{S}, \\
F_{A} & =F_{G}
\end{aligned}
$$

is equivalent to (46). Denote $G^{\prime}=A^{-}+A^{-} B S^{-} C A^{-}$. By $F_{A} B=$ $0, B E_{D}=0$, and $F_{S}=F_{D}$, a simple computation shows that

$$
\begin{aligned}
G G^{\prime}= & \left(A-B D^{-} C\right)\left(A^{-}+A^{-} B S^{-} C A^{-}\right) \\
= & A A^{-}+A A^{-} B S^{-} C A^{-}-B D^{-} C A^{-} \\
& -B D^{-} D S^{-} C A^{-}+B D^{-} S S^{-} C A^{-}=A A^{-} .
\end{aligned}
$$

Similarly, by $F_{D} C=0, C E_{A}=0$, and $E_{S}=E_{D}$, we get $G^{\prime} G=$ $A^{-} A$. Moreover, $G G^{\prime} G=G$ and $G^{\prime} G G^{\prime}=G^{\prime}$. Thus, $G^{\prime}=G^{\dagger}$ and $E_{A}=E_{G}$ and $F_{A}=F_{G}$. Therefore, (51) is equivalent to (46).
Corollary 14 (see [9]). Let $M, S$, and $G$ be defined by (3), (5), and (6), respectively. Then

$$
\begin{aligned}
M^{\dagger} & =\left[\begin{array}{cc}
A^{-}+A^{-} B S^{-} C A^{-} & -A^{-} B S^{-} \\
-S^{-} C A^{-} & S^{-}
\end{array}\right] \\
& =\left[\begin{array}{cc}
G^{-} & -G^{-} B D^{-} \\
-D^{-} C G^{-} & D^{-}+D^{-} C G^{-} B D^{-}
\end{array}\right]
\end{aligned}
$$

if and only if

$$
\begin{gathered}
A^{-}=A^{\dagger}, \\
D^{-}=D^{\dagger}, \\
S^{-}=S^{\dagger}, \\
G^{-}=G^{\dagger}, \\
F_{A} B=0, \\
F_{D} C=0, \\
F_{S} C=0, \\
C E_{A}=0, \\
B E_{D}=0, \\
B E_{S}=0 .
\end{gathered}
$$

Proof. By Corollary 14 from [9], it is easy to conclude that (54) is equivalent to (46).

Corollary 15. Let $M, S$, and $G$ be defined by (3), (5), and (6), respectively. Then

$$
\begin{aligned}
M^{\dagger} & =\left[\begin{array}{cc}
A^{-}+A^{-} B S^{-} C A^{-} & -A^{-} B S^{-} \\
-S^{-} C A^{-} & S^{-}
\end{array}\right] \\
& =\left[\begin{array}{cc}
G^{-} & -G^{-} B D^{-} \\
-D^{-} C G^{-} & D^{-}+D^{-} C G^{-} B D^{-}
\end{array}\right]
\end{aligned}
$$

if and only if

$$
\begin{aligned}
A^{-} & =A^{\dagger}, \\
D^{-} & =D^{\dagger}, \\
S^{-} & =S^{\dagger}, \\
G^{-} & =G^{\dagger}, \\
F_{A} B & =0, \\
F_{D} C & =0, \\
C E_{A} & =0, \\
B E_{D} & =0, \\
E_{A} & =E_{G}, \\
F_{A} & =F_{G} .
\end{aligned}
$$


Corollary 16. Let $M, S$, and $G$ be defined by (3), (5), and (6), respectively. Then

$$
\begin{aligned}
M^{\dagger} & =\left[\begin{array}{cc}
A^{-}+A^{-} B S^{-} C A^{-} & -A^{-} B S^{-} \\
-S^{-} C A^{-} & S^{-}
\end{array}\right] \\
& =\left[\begin{array}{cc}
G^{-} & -G^{-} B D^{-} \\
-D^{-} C G^{-} & D^{-}+D^{-} C G^{-} B D^{-}
\end{array}\right]
\end{aligned}
$$

if and only if

$$
\begin{aligned}
A^{-} & =A^{\dagger}, \\
D^{-} & =D^{\dagger}, \\
S^{-} & =S^{\dagger}, \\
G^{-} & =G^{\dagger}, \\
F_{A} B & =0, \\
F_{D} C & =0, \\
F_{G} B & =0, \\
C E_{A} & =0, \\
B E_{D} & =0, \\
C E_{G} & =0 .
\end{aligned}
$$

Theorem 17. Let $M, S$, and $G$ be defined by (3), (5), and (6), respectively. Then

$$
\begin{aligned}
M^{\#} & =\left[\begin{array}{cc}
A^{-}+A^{-} B S^{-} C A^{-} & -A^{-} B S^{-} \\
-S^{-} C A^{-} & S^{-}
\end{array}\right] \\
& =\left[\begin{array}{cc}
G^{-} & -G^{-} B D^{-} \\
-D^{-} C G^{-} & D^{-}+D^{-} C G^{-} B D^{-}
\end{array}\right]
\end{aligned}
$$

if and only if one of the following conditions holds.

(i)

$$
\begin{gathered}
A^{-}=A^{\#}, \\
D^{-}=D^{\#}, \\
S^{-}=S^{\#}, \\
G^{-}=G^{\#}, \\
F_{A} B=0, \\
F_{D} C=0, \\
F_{S} C=0, \\
C E_{A}=0, \\
B E_{D}=0, \\
B E_{S}=0 .
\end{gathered}
$$

(ii)

$$
\begin{aligned}
A^{-} & =A^{\#}, \\
D^{-} & =D^{\#}, \\
S^{-} & =S^{\#}, \\
G^{-} & =G^{\#}, \\
F_{A} B & =0, \\
F_{D} C & =0, \\
F_{G} B & =0, \\
C E_{A} & =0, \\
B E_{D} & =0, \\
C E_{G} & =0 .
\end{aligned}
$$

Proof. (i) By Lemma 8,

$$
M^{\#}=\left[\begin{array}{cc}
A^{-}+A^{-} B S^{-} C A^{-} & -A^{-} B S^{-} \\
-S^{-} C A^{-} & S^{-}
\end{array}\right]
$$

if and only if

$$
\begin{aligned}
A^{-} & =A^{\#}, \\
S^{-} & =S^{\#}, \\
F_{A} B & =0, \\
F_{S} C & =0, \\
C E_{A} & =0, \\
B E_{S} & =0 .
\end{aligned}
$$

Similarly,

$$
M^{\#}=\left[\begin{array}{cc}
G^{-} & -G^{-} B D^{-} \\
-D^{-} C G^{-} & D^{-}+D^{-} C G^{-} B D^{-}
\end{array}\right]
$$

if and only if

$$
\begin{gathered}
D^{-}=D^{\#,} \\
G^{-}=G^{\#}, \\
F_{G} B=0, \\
F_{D} C=0, \\
C E_{G}=0, \\
B E_{D}=0 .
\end{gathered}
$$


Since the group inverse is unique if it exists, combining (63) and (65), we get

$$
\begin{aligned}
M^{\#} & =\left[\begin{array}{cc}
A^{-}+A^{-} B S^{-} C A^{-} & -A^{-} B S^{-} \\
-S^{-} C A^{-} & S^{-}
\end{array}\right] \\
& =\left[\begin{array}{cc}
G^{-} & -G^{-} B D^{-} \\
-D^{-} C G^{-} & D^{-}+D^{-} C G^{-} B D^{-}
\end{array}\right]
\end{aligned}
$$

if and only if

$$
\begin{aligned}
A^{-} & =A^{\#}, \\
D^{-} & =D^{\#}, \\
S^{-} & =S^{\#}, \\
G^{-} & =G^{\#}, \\
F_{A} B & =0, \\
F_{G} B & =0, \\
F_{S} C & =0, \\
F_{D} C & =0, \\
B E_{S} & =0, \\
C E_{A} & =0, \\
B E_{D} & =0, \\
C E_{G} & =0 .
\end{aligned}
$$

Now, we only need to prove (67) is equivalent to (60). Denote $G^{\prime}=A^{-}+A^{-} B S^{-} C A^{-}$. If (60) holds, then

$$
\begin{aligned}
G G^{\prime}= & \left(A-B D^{-} C\right)\left(A^{-}+A^{-} B S^{-} C A^{-}\right) \\
= & A A^{-}+A A^{-} B S^{-} C A^{-}-B D^{-} C A^{-} \\
& -B D^{-} C A^{-} B S^{-} C A^{-} \\
= & A A^{-}+B S^{-} C A^{-}-B D^{-} C A^{-} \\
& -B D^{-}(D-S) S^{-} C A^{-}=A A^{-}, \\
G^{\prime} G= & \left(A^{-}+A^{-} B S^{-} C A^{-}\right)\left(A-B D^{-} C\right) \\
= & A^{-} A-A^{-} B D^{-} C-A^{-} B S^{-} C A^{-} A \\
& -A^{-} B S^{-} C A^{-} B D^{-} C \\
= & A^{-} A-A^{-} B D^{-} C-A^{-} B S^{-} C \\
& -A^{-} B S^{-}(D-S) D^{-} C=A^{-} A .
\end{aligned}
$$

Now, it is easy to get

$$
\begin{aligned}
G G^{\prime} G & =A A^{-}\left(A-B D^{-} C\right)=A-B D^{-} C=G, \\
G^{\prime} G G^{\prime} & =A^{-} A\left(A^{-}+A^{-} B S^{-} C A^{-}\right) \\
& =A^{-}+A^{-} B S^{-} C A^{-}=G^{\prime}
\end{aligned}
$$

and $G G^{\prime}=G^{\prime} G$. Thus, $G^{\prime}=G^{\#}$. Hence, $G^{\#} G=A^{-} A$ and $G G^{\#}=A A^{-}$. Now, we get $F_{A} B=F_{G} B=0$ and $C E_{A}=$ $C E_{G}=0$, which means (60) implies (67). Obviously, (67) implies (60). Thus, (67) is equivalent to (60).

(ii) The proof is similar to the proof of (i).

\section{Representations of Drazin Inverse in terms of Banachiewicz-Schur Forms}

The Drazin inverse of a square matrix has various applications in singular differential equations and singular difference equations, Markov chains, and iterative methods. Actually, in 1979 Campbell and Meyer [13] posed the problem of finding an explicit representation for the Drazin inverse of a complex block matrix $M$, in terms of its blocks. No formula for $M^{D}$ has yet been offered without any restrictions upon the blocks. Many papers have considered this open problem and each of them offered a formula for the Drazin inverse and specific conditions for the $2 \times 2$ block matrix $M$ (see $[9,11,12,14-22]$ ).

Wei [23] proved that $M^{D}$ can be expressed by $\left[\begin{array}{c}A^{D}+A^{D} B S^{D} C A^{D}-A^{D} B S^{D} \\ -S^{D} C A^{D}\end{array}\right]$ if $A^{\pi} C=0, B A^{\pi}=0, C S^{\pi}=0$, $S^{\pi} B=0$, and $D S^{\pi}=0$, where $A^{\pi}=I-A A^{D}$ and $S^{\pi}=I-S S^{D}$.

Cvetković-Ilić [9] generalized the result from [23] and gave the representation of $M^{D}$ under simpler conditions than in [23].

Sheng and Chen [12] proved that, under the conditions $A^{\pi} B=0, B D^{\pi}=0, C A^{\pi}=0, D^{\pi} C=0$, and $S^{\pi}=D^{\pi}=0, M^{D}$ can be represented by both of Banachiewicz-Schur forms.

In the following, we will present conditions more general than those in $[9,12,23]$ which ensure the representation of $M^{D}$ by the Banachiewicz-Schur form.

Theorem 18. Let $M$ be defined by (3) and $S=D-C A^{D} B$ be the generalized Schur complement of $A$ in $M$. If

$$
\begin{aligned}
& A^{D} B S^{\pi}=0, \\
& S^{D} C A^{\pi}=0, \\
& A^{\pi} B S^{D}=0, \\
& S^{\pi} C A^{D}=0,
\end{aligned}
$$

then

$$
M^{D}=\left[\begin{array}{cc}
A^{D}+A^{D} B S^{D} C A^{D} & -A^{D} B S^{D} \\
-S^{D} C A^{D} & S^{D}
\end{array}\right] .
$$

Proof. Denote the right side of (71) by $X$. By simple computations, we show that

$M X$

$$
=\left[\begin{array}{cc}
A A^{D}-\left(I-A A^{D}\right) B S^{D} C A^{D} & \left(I-A A^{D}\right) B S^{D} \\
\left(I-S S^{D}\right) C A^{D} & S S^{D}
\end{array}\right],
$$


$X M$

$$
=\left[\begin{array}{cc}
A^{D} A-A^{D} B S^{D} C\left(I-A^{D} A\right) & A^{D} B\left(I-S^{D} S\right) \\
S^{D} C\left(I-A^{D} A\right) & S^{D} S
\end{array}\right] .
$$

Since $A^{D} B S^{\pi}=0, S^{D} C A^{\pi}=0, A^{\pi} B S^{D}=0$, and $S^{\pi} C A^{D}=0$, (72) reduce to

$$
\begin{aligned}
& M X=\left[\begin{array}{cc}
A A^{D} & 0 \\
0 & S S^{D}
\end{array}\right], \\
& X M=\left[\begin{array}{cc}
A^{D} A & 0 \\
0 & S^{D} S
\end{array}\right] .
\end{aligned}
$$

Obviously, $M X=X M$. On the other hand,

$X M X$

$$
\begin{aligned}
& =\left[\begin{array}{cc}
A^{D} A & 0 \\
0 & S^{D} S
\end{array}\right]\left[\begin{array}{cc}
A^{D}+A^{D} B S^{D} C A^{D} & -A^{D} B S^{D} \\
-S^{D} C A^{D} & S^{D}
\end{array}\right] \\
& =\left[\begin{array}{cc}
A^{D}+A^{D} B S^{D} C A^{D} & -A^{D} B S^{D} \\
-S^{D} C A^{D} & S^{D}
\end{array}\right]=X .
\end{aligned}
$$

Now, we need to prove that $M-M^{2} N$ is a nilpotent matrix. Since

$$
M-M^{2} N=\left[\begin{array}{cc}
\left(A-\left(I-A A^{D}\right) B S^{D} C\right)\left(I-A^{D} A\right) & \left(I-A A^{D}\right) B\left(I-S^{D} S\right) \\
\left(I-S S^{D}\right) C\left(I-A^{D} A\right) & S\left(I-S S^{D}\right)
\end{array}\right],
$$

by $A^{D} B S^{\pi}=0, S^{D} C A^{\pi}=0$, we get

$$
M-M^{2} N=\left[\begin{array}{cc}
A\left(I-A^{D} A\right) & 0 \\
0 & S\left(I-S S^{D}\right)
\end{array}\right],
$$

which is nilpotent and $\operatorname{ind}\left(M-M^{2} N\right) \geqslant \max \{\operatorname{ind}(A)$, ind $(S)\}$.

Corollary 19 (see [9]). Let $M$ be given by (3) and $S=D-$ $C A^{D} B$ be the generalized Schur complement of $A$ in $M$. If

$$
\begin{aligned}
& A^{D} B S^{\pi}=0, \\
& S^{D} C A^{\pi}=0, \\
& A^{\pi} B S^{D}=0, \\
& S^{\pi} C A^{D}=0
\end{aligned}
$$

and if there exists a nonnegative integer $k$ such that $M^{k} P=0$, then

$$
M^{D}=\left[\begin{array}{cc}
A^{D}+A^{D} B S^{D} C A^{D} & -A^{D} B S^{D} \\
-S^{D} C A^{D} & S^{D}
\end{array}\right],
$$

where $P=\left[\begin{array}{cc}A^{\pi} & 0 \\ 0 & S^{\pi}\end{array}\right]$.

Corollary 20 (see [23]). Let $M$ be given by (3) and $S=D-$ $C A^{D} B$ be the generalized Schur complement of $A$ in $M$. If

$$
\begin{aligned}
& A^{\pi} B=0, \\
& B S^{\pi}=0, \\
& C A^{\pi}=0,
\end{aligned}
$$

$$
\begin{aligned}
& S^{\pi} C=0, \\
& D S^{\pi}=0,
\end{aligned}
$$

then

$$
M^{D}=\left[\begin{array}{cc}
A^{D}+A^{D} B S^{D} C A^{D} & -A^{D} B S^{D} \\
-S^{D} C A^{D} & S^{D}
\end{array}\right] .
$$

Theorem 21. Let $M$ be defined as in (3) and $G=A-B D^{D} C$ be the generalized Schur complement of $D$ in $M$. If

$$
\begin{aligned}
& D^{D} C G^{\pi}=0, \\
& G^{D} B D^{\pi}=0, \\
& G^{\pi} B D^{D}=0, \\
& D^{\pi} C G^{D}=0,
\end{aligned}
$$

then

$$
M^{D}=\left[\begin{array}{cc}
G^{D} & -G^{D} B D^{D} \\
-D^{D} C G^{D} & D^{D}+D^{D} C G^{D} B D^{D}
\end{array}\right] .
$$

Proof. The proof is analogous to that of Theorem 18.

Theorem 22. Let $M$ be defined by (3) and $S=D-C A^{D} B$, $G=A-B D^{D} C$ be the generalized Schur complement of $A$ in $M$ and $D$ in $M$, respectively. If any of the conditions hold

(i) $A^{\pi} B=0, C A^{\pi}=0, B D^{\pi}=0, D^{\pi} C=0, S^{\pi} C A^{D}=0$, $A^{D} B S^{\pi}=0$,

(ii) $A^{\pi} B=0, C A^{\pi}=0, B D^{\pi}=0, D^{\pi} C=0, G^{\pi} B D^{D}=0$, $D^{D} C G^{\pi}=0$, 
then

$$
\begin{aligned}
M^{D} & =\left[\begin{array}{cc}
A^{D}+A^{D} B S^{D} C A^{D} & -A^{D} B S^{D} \\
-S^{D} C A^{D} & S^{D}
\end{array}\right] \\
& =\left[\begin{array}{cc}
G^{D} & -G^{D} B D^{D} \\
-D^{D} C G^{D} & D^{D}+D^{D} C G^{D} B D^{D}
\end{array}\right] .
\end{aligned}
$$

Proof. (i) Denote $X=A^{D}+A^{D} B S^{D} C A^{D}$. Firstly, we prove $G^{D}=A^{D}+A^{D} B S^{D} C A^{D}$. It is easy to see

$$
\begin{aligned}
G X= & \left(A-B D^{D} C\right)\left(A^{D}+A^{D} B S^{D} C A^{D}\right) \\
= & A A^{D}+A A^{D} B S^{D} C A^{D}-B D^{D} C A^{D} \\
& -B D^{D} C A^{D} B S^{D} C A^{D} \\
= & A A^{D}+A A^{D} B S^{D} C A^{D}-B D^{D} C A^{D} \\
& -B D^{D}(D-S) S^{D} C A^{D} \\
= & A A^{D}+A A^{D} B S^{D} C A^{D}-B D^{D} C A^{D} \\
& -B D^{D} D S^{D} C A^{D}+B D^{D} S S^{D} C A^{D}=A A^{D}
\end{aligned}
$$

$$
\begin{aligned}
X G= & \left(A^{D}+A^{D} B S^{D} C A^{D}\right)\left(A-B D^{D} C\right) \\
= & A^{D} A+A^{D} B D^{D} C-A^{D} B S^{D} C A^{D} A \\
& -A^{D} B S^{D} C A^{D} B D^{D} C \\
= & A^{D} A+A^{D} B D^{D} C-A^{D} B S^{D} C A^{D} A \\
& -A^{D} B S^{D}(D-S) D^{D} C \\
= & A^{D} A+A^{D} B D^{D} C-A^{D} B S^{D} C A^{D} A \\
& -A^{D} B S^{D} D D^{D} C+A^{D} B S^{D} S D^{D} C=A^{D} A
\end{aligned}
$$

by $C A^{\pi}=0, D^{\pi} C=0$, and $A^{D} B S^{\pi}=0$. Thus, $G X=X G$. Moreover,

$$
\begin{aligned}
X G X & =A^{D} A\left(A^{D}+A^{D} B S^{D} C A^{D}\right) \\
& =\left(A^{D}+A^{D} B S^{D} C A^{D}\right)=X, \\
G^{k} X G & =G^{k} A^{D} A=G^{k-1}\left(A-B D^{D} C\right) A^{D} A \\
& =G^{k-1}\left(A-B D^{D} C\right)=G^{k},
\end{aligned}
$$

by using $C A^{\pi}=0$. Therefore, $G G^{D}=A A^{D}$. Since $A^{\pi} B=0$ and $C A^{\pi}=0$, we get $G^{\pi} B=0, C G^{\pi}=0$. Now, we can deduce that

$$
M^{D}=\left[\begin{array}{cc}
A^{D}+A^{D} B S^{D} C A^{D} & -A^{D} B S^{D} \\
-S^{D} C A^{D} & S^{D}
\end{array}\right],
$$

by $A^{D} B S^{\pi}=0, C A^{\pi}=0, A^{\pi} B=0$, and $S^{\pi} C A^{D}=0$ and

$$
M^{D}=\left[\begin{array}{cc}
G^{D} & -G^{D} B D^{D} \\
-D^{D} C G^{D} & D^{D}+D^{D} C G^{D} B D^{D}
\end{array}\right],
$$

by $C G^{\pi}=0, B D^{\pi}=0, G^{\pi} B=0$, and $D^{\pi} C=0$. Then,

$$
\begin{aligned}
M^{D} & =\left[\begin{array}{cc}
A^{D}+A^{D} B S^{D} C A^{D} & -A^{D} B S^{D} \\
-S^{D} C A^{D} & S^{D}
\end{array}\right] \\
& =\left[\begin{array}{cc}
G^{D} & -G^{D} B D^{D} \\
-D^{D} C G^{D} & D^{D}+D^{D} C G^{D} B D^{D}
\end{array}\right]
\end{aligned}
$$

follows by the uniqueness of the Drazin inverse.

(ii) Similarly, we denote $Y=D^{D}+D^{D} C G^{D} B D^{D}$. In the same way, we can easily get $S Y=D D^{D}$ by $C A^{\pi}=0, B D^{\pi}=0$, $D^{\pi} C G^{D}=0$, and $Y S=D^{D} D$ by $A^{\pi} B=0, C G^{\pi}=0$, and $G^{\pi} B D^{D}=0$. Since

$$
\begin{aligned}
Y S Y & =D^{D} D\left(D^{D}+D^{D} C G^{D} B D^{D}\right) \\
& =D^{D}+D^{D} C G^{D} B D^{D}=Y, \\
S^{k} Y S & =S^{k-1}\left(D-C A^{D} B\right) D^{D} D=S^{k-1}\left(D-C A^{D} B\right) \\
& =S^{k},
\end{aligned}
$$

by using $B D^{\pi}=0$, we obtain $S^{D}=D^{D}+D^{D} C G^{D} B D^{D}$. Thus, we have $S S^{D}=D D^{D}$ which implies $B S^{\pi}=0$ and $S^{\pi} C=0$, where we used $B D^{\pi}=0$ and $D^{\pi} C=0$. Now, by $B S^{\pi}=0$, $S^{\pi} C=0, A^{\pi} B=0$, and $C A^{\pi}=0$, we get

$$
M^{D}=\left[\begin{array}{cc}
A^{D}+A^{D} B S^{D} C A^{D} & -A^{D} B S^{D} \\
S^{D} C A^{D} & S^{D}
\end{array}\right],
$$

and by $D^{\pi} C=0, B D^{\pi}=0, G^{\pi} B D^{D}=0$, and $D^{D} C G^{\pi}=0$, we have

$$
M^{D}=\left[\begin{array}{cc}
G^{D} & -G^{D} B D^{D} \\
-D^{D} C G^{D} & D^{D}+D^{D} C G^{D} B D^{D}
\end{array}\right] .
$$

Therefore,

$$
\begin{aligned}
M^{D} & =\left[\begin{array}{cc}
A^{D}+A^{D} B S^{D} C A^{D} & -A^{D} B S^{D} \\
-S^{D} C A^{D} & S^{D}
\end{array}\right] \\
& =\left[\begin{array}{cc}
G^{D} & -G^{D} B D^{D} \\
-D^{D} C G^{D} & D^{D}+D^{D} C G^{D} B D^{D}
\end{array}\right] .
\end{aligned}
$$


Corollary 23 (see [12]). Let $M$ be defined by (3) and $S=D$ $C A^{D} B, G=A-B D^{D} C$ be the generalized Schur complement of $A$ in $M$ and $D$ in $M$, respectively. If

$$
\begin{aligned}
A^{\pi} B & =0, \\
B D^{\pi} & =0, \\
C A^{\pi} & =0, \\
D^{\pi} C & =0, \\
S^{\pi} & =D^{\pi},
\end{aligned}
$$

then

$$
\begin{aligned}
M^{D} & =\left[\begin{array}{cc}
A^{D}+A^{D} B S^{D} C A^{D} & -A^{D} B S^{D} \\
-S^{D} C A^{D} & S^{D}
\end{array}\right] \\
& =\left[\begin{array}{cc}
G^{D} & -G^{D} B D^{D} \\
-D^{D} C G^{D} & D^{D}+D^{D} C G^{D} B D^{D}
\end{array}\right] .
\end{aligned}
$$

Proof. According to the proof of Theorem 22(ii), we have $S^{D}=Y=D^{D}+D^{D} C G^{D} B D^{D}$ which implies $S S^{D}=D D^{D}$ by $S Y=D D^{D}$. Now, it is sufficient to conclude that (95) holds.

\section{Quotient Property and the First \\ Sylvester Identity in terms of the Generalized Schur Complement}

Crabtree and Haynsworth in [18] showed a quotient formula for Schur complement of a matrix. After that, the formula was extended by Ostrowski in 1971 [24] and in 1973 [19].

Let

$$
\begin{aligned}
Q & =\left[\begin{array}{lll}
A & B & E \\
C & D & F \\
G & H & J
\end{array}\right], \\
M & =\left[\begin{array}{ll}
A & B \\
C & D
\end{array}\right], \\
M_{2} & =\left[\begin{array}{ll}
B & E \\
D & F
\end{array}\right], \\
M_{3} & =\left[\begin{array}{ll}
D & F \\
H & J
\end{array}\right], \\
M_{4} & =\left[\begin{array}{ll}
C & D \\
G & H
\end{array}\right] .
\end{aligned}
$$

If $Q$ and $M$ are square and nonsingular, then

$$
\begin{aligned}
\left(\frac{Q}{M}\right) & =\left(\frac{(Q / A)}{(M / A)}\right) \\
& =\left(\frac{\left[\begin{array}{ll}
A & E \\
G & J
\end{array}\right]}{A}\right)-\left(\frac{\left[\begin{array}{ll}
A & B \\
G
\end{array}\right]}{A}\right)\left(\frac{M}{A}\right)^{-1}\left(\frac{\left[\begin{array}{cc}
A & E \\
C & F
\end{array}\right]}{A}\right) .
\end{aligned}
$$

This is the so-called quotient property which is proven by Crabtree and Haynsworth in [18].

The first Sylvester identity is that

$$
\begin{aligned}
\left(\frac{Q}{M_{3}}\right) & =\left(\frac{(Q / D)}{\left(M_{3} / D\right)}\right) \\
& =\left(\frac{M}{D}\right)-\left(\frac{M_{2}}{D}\right)\left(\frac{M_{3}}{D}\right)^{-1}\left(\frac{M_{4}}{D}\right),
\end{aligned}
$$

where $M_{3}$ and $D$ are nonsingular.

Sheng and Chen [12] studied the quotient properties and the first Sylvester identity with respect to MP, group, and Drazin inverses. Cvetković-Ilić in [9] derived the properties under conditions different than those used in [12].

In this section, we will give the quotient properties and the first Sylvester identity of MP, group, and Drazin inverses under the conditions which are much simpler than those of $[9,12]$. Notice that the proofs of the following results are almost identical to the proofs of the corresponding results from [12]. We will state the theorems without proofs.

We first give the quotient properties based on MP, group, and Drazin inverses. For convenience, we denote

$$
\begin{aligned}
& \left(\frac{M}{A}\right)_{p}=D-C A^{\dagger} B, \\
& \left(\frac{M}{A}\right)_{g}=D-C A^{\#} B, \\
& \left(\frac{M}{A}\right)_{d}=D-C A^{D} B, \\
& \left(\frac{M}{D}\right)_{p}=A-B D^{\dagger} C, \\
& \left(\frac{M}{D}\right)_{g}=A-B D^{\#} C, \\
& \left(\frac{M}{D}\right)_{d}=A-B D^{D} C .
\end{aligned}
$$

Theorem 24. Let $Q=\left[\begin{array}{lll}A & B & E \\ C & D & F \\ G & H & J\end{array}\right]$ and $M=\left[\begin{array}{ll}A & B \\ C & D\end{array}\right]$. If $M$ satisfies the conditions of Corollary 15, then

$$
\left(\frac{Q}{M}\right)_{p}=\left(\frac{(Q / A)_{p}}{(M / A)_{p}}\right)_{p} .
$$

Theorem 25. Let $Q=\left[\begin{array}{lll}A & B & E \\ C & D & F \\ G & H & J\end{array}\right]$ and $M=\left[\begin{array}{ll}A & B \\ C & D\end{array}\right]$. If $M$ satisfies the conditions of Theorem 11(i) or (ii), then

$$
\left(\frac{Q}{M}\right)_{g}=\left(\frac{(Q / A)_{g}}{(M / A)_{g}}\right)_{g} .
$$

Theorem 26. Let $Q=\left[\begin{array}{ccc}A & B & E \\ C & D \\ G & F & J\end{array}\right]$ and $M=\left[\begin{array}{ll}A & B \\ C & D\end{array}\right]$. If $M$ satisfies the conditions of Theorem 18, then

$$
\left(\frac{Q}{M}\right)_{d}=\left(\frac{(Q / A)_{d}}{(M / A)_{d}}\right)_{d} .
$$


The first Sylvester identity with respect to MP, group, and Drazin inverses is expressed as follows.

Theorem 27. Let $Q, M, M_{2}, M_{3}$, and $M_{4}$ be the forms of (97). If $M_{3}$ satisfies the conditions of Corollary 15 , then

$$
\begin{aligned}
\left(\frac{\mathrm{Q}}{M_{3}}\right)_{p} & =\left(\frac{(\mathrm{Q} / D)_{p}}{\left(M_{3} / D\right)_{p}}\right)_{p} \\
& =\left(\frac{M}{D}\right)_{p}-\left(\frac{M_{2}}{D}\right)_{p}\left(\frac{M_{3}}{D}\right)_{p}^{\dagger}\left(\frac{M_{4}}{D}\right)_{p}
\end{aligned}
$$

Theorem 28. Let $Q, M, M_{2}, M_{3}$, and $M_{4}$ be the forms of (97). If $M_{3}$ satisfies the conditions of Theorem 11(i) or (ii), then

$$
\begin{aligned}
\left(\frac{Q}{M_{3}}\right)_{g} & =\left(\frac{(Q / D)_{g}}{\left(M_{3} / D\right)_{g}}\right)_{g} \\
& =\left(\frac{M}{D}\right)_{g}-\left(\frac{M_{2}}{D}\right)_{g}\left(\frac{M_{3}}{D}\right)_{g}^{\#}\left(\frac{M_{4}}{D}\right)_{g} .
\end{aligned}
$$

Theorem 29. Let $Q, M, M_{2}, M_{3}$, and $M_{4}$ be the forms of (97). If $M_{3}$ satisfies the conditions of Theorem 18, then

$$
\begin{aligned}
\left(\frac{Q}{M_{3}}\right)_{d} & =\left(\frac{(Q / D)_{d}}{\left(M_{3} / D\right)_{d}}\right)_{d} \\
& =\left(\frac{M}{D}\right)_{d}-\left(\frac{M_{2}}{D}\right)_{d}\left(\frac{M_{3}}{D}\right)_{d}^{D}\left(\frac{M_{4}}{D}\right)_{d}
\end{aligned}
$$

\section{Applications of the Generalized Schur Complement}

The Schur complements and generalized Schur complements were shown playing an important role in various applied mathematical settings, such as statistics, matrix theory, electrical network theory, discrete-time regulator problem, sophisticated techniques, and many other fields.

In this section, we will give an application of the generalized Schur complement in the solution of a linear system. Using the generalized Schur complement, we can split a larger system into two small linear systems by the following steps.

Let

$$
M x=y
$$

be a linear system. Applying the block Gaussian elimination to the system, we have

$$
\left[\begin{array}{cc}
A & B \\
0 & D-C A^{\dagger} B
\end{array}\right]\left[\begin{array}{l}
x_{1} \\
x_{2}
\end{array}\right]=\left[\begin{array}{c}
y_{1} \\
y_{2}-C A^{\dagger} y_{1}
\end{array}\right] .
$$

Hence, we get

$$
\begin{aligned}
A x_{1}+B x_{2} & =y_{1} \\
S x_{2} & =y_{2}-C A^{\dagger} y_{1}
\end{aligned}
$$

that is,

$$
\begin{aligned}
& A x_{1}=y_{1}-B x_{2}, \\
& S x_{2}=y_{2}-C A^{\dagger} y_{1} .
\end{aligned}
$$

Now, the solution of system (107) can be obtained by the two small linear systems above. In this case, the operation can be significantly simplified. We will also notice that the MoorePenrose inverse of $A$ can be replaced by other generalized inverses, such as the group inverse, the Drazin inverse, and the generalized inverse of $A$ or even the ordinary inverse $A^{-1}$.

In the following, we will give the group inverse solutions of the linear system.

Theorem 30. Let

$$
M x=y
$$

be a linear system. Suppose $M$ satisfies all the conditions of Theorem 17, partitioning $x$ and $y$ as

$$
\begin{aligned}
& x=\left[\begin{array}{l}
x_{1} \\
x_{2}
\end{array}\right], \\
& y=\left[\begin{array}{l}
y_{1} \\
y_{2}
\end{array}\right],
\end{aligned}
$$

which have appropriate sizes with $M$. If $y \in R(M)$, then the solution $x=M^{\#} y$ of linear system (111) can be expressed as

$$
\begin{aligned}
& x_{1}=A^{\#}\left(y_{1}-B x_{2}\right), \\
& x_{2}=S^{\#}\left(y_{2}-C A^{\#} y_{1}\right),
\end{aligned}
$$

where $S=D-C A^{\#} B$.

Proof. Since $y \in R(M)$, we conclude that $x=M^{\#} y$ is the solution of linear system (111). By Theorem 17, we can get the following:

$$
\begin{gathered}
x=M^{\#} y=\left[\begin{array}{cc}
A^{\#}+A^{\#} B S^{\#} C A^{\#} & -A^{\#} B S^{\#} \\
-S^{\#} C A^{\#} & S^{\#}
\end{array}\right]\left[\begin{array}{l}
y_{1} \\
y_{2}
\end{array}\right] \\
=\left[\begin{array}{c}
A^{\#} y_{1}+A^{\#} B S^{\#} C A^{\#} y_{1}-A^{\#} B S^{\#} y_{2} \\
S^{\#}\left(y_{2}-C A^{\#} y_{1}\right)
\end{array}\right]=\left[\begin{array}{l}
x_{1} \\
x_{2}
\end{array}\right] .
\end{gathered}
$$

Now, it is easy to see the solution $x=M^{\#} y$ can be expressed as

$$
\begin{aligned}
& x_{1}=A^{\#}\left(y_{1}-B x_{2}\right), \\
& x_{2}=S^{\#}\left(y_{2}-C A^{\#} y_{1}\right),
\end{aligned}
$$

which is also the group inverse solutions of the two small linear systems of (110), respectively.

\section{Competing Interests}

The authors declare that they have no competing interests. 


\section{Acknowledgments}

This work was supported by the National Natural Science Foundation of China (11361009). The third author is supported by Grant no. 174007 of the Ministry of Education, Science and Technological Development, Serbia.

\section{References}

[1] I. Schur, "Potenzreihn in innern des heitskreises," Journal für die Reine und Angewandte Mathematik, vol. 147, pp. 205-232, 1917.

[2] T. Ando, "Generalized Schur complements," Linear Algebra and Its Applications, vol. 27, pp. 173-186, 1979.

[3] D. Carlson, "What are Schur complements, anyway?" Linear Algebra and Its Applications, vol. 74, pp. 257-275, 1986.

[4] D. Carlson, E. Haynsworth, and T. Markham, "A generalization of the Schur complement by means of the Moore-Penrose inverse," SIAM Journal on Applied Mathematics, vol. 26, pp. 169$175,1974$.

[5] C.-K. Li and R. Mathias, "Extremal characterizations of the Schur complement and resulting inequalities," SIAM Review, vol. 42, no. 2, pp. 233-246, 2000.

[6] G. Corach, A. Maestripieri, and D. Stojanoff, "Oblique projections and Schur complements," Acta Scientiarum Mathematicarum, vol. 67, pp. 439-459, 2001.

[7] G. Corach, A. Maestripieri, and D. Stojanoff, "Generalized Schur complements and oblique projections," Linear Algebra and Its Applications, vol. 341, no. 1-3, pp. 259-272, 2002.

[8] R. W. Cottle, "Manifestations of the Schur complement," Linear Algebra and its Applications, vol. 8, no. 3, pp. 189-211, 1974.

[9] D. S. Cvetković-Ilić, "Expression of the Drazin and MP-inverse of partitioned matrix and quotient identity of generalized Schur complement," Applied Mathematics and Computation, vol. 213, no. 1, pp. 18-24, 2009.

[10] J. K. Baksalary and G. P. Styan, "Generalized inverses of partitioned matrices in Banachiewicz-Schur form," Linear Algebra and Its Applications, vol. 354, pp. 41-47, 2002.

[11] J. Benítez and N. Thome, "The generalized Schur complement in group inverses and $(k+1)$-potent matrices," Linear and Multilinear Algebra, vol. 54, no. 6, pp. 405-413, 2006.

[12] X. Sheng and G. Chen, "Some generalized inverses of partition matrix and quotient identity of generalized Schur complement," Applied Mathematics and Computation, vol. 196, no. 1, pp. 174184,2008

[13] S. L. Campbell and C. D. Meyer, Generalized Inverse of Linear Transformations, Pitman, London, UK, 1979.

[14] N. Castro-González, E. Dopazo, and J. Robles, "Formulas for the Drazin inverse of special block matrices," Applied Mathematics and Computation, vol. 174, no. 1, pp. 252-270, 2006.

[15] D. S. Cvetković-Ilić, "A note on the representation for the Drazin inverse of $2 \times 2$ block matrices," Linear Algebra and its Applications, vol. 429, no. 1, pp. 242-248, 2008.

[16] D. S. Cvetković-Ilić, J. Chen, and Z. Xu, "Explicit representations of the Drazin inverse of block matrix and modified matrix," Linear and Multilinear Algebra, vol. 57, no. 4, pp. 355364, 2009.

[17] D. S. Djordjević and P. S. Stanimirović, "On the generalized Drazin inverse and generalized resolvent," Czechoslovak Mathematical Journal, vol. 51, no. 3, pp. 617-634, 2001.
[18] D. E. Crabtree and E. V. Haynsworth, "An identity for the Schur complement of a matrix," Proceedings of the American Mathematical Society, vol. 22, pp. 364-366, 1969.

[19] A. M. Ostrowski, “On Schur's complement," Journal of Combinatorial Theory. Series A, vol. 14, pp. 319-323, 1973.

[20] R. E. Hartwig and J. M. Shoaf, "Group inverses and Drazin inverses of bidiagonal and triangular Toeqlitz matrices," Journal of the Australian Mathematical Society, vol. 24, no. 1, pp. 10-14, 1977.

[21] D. S. Cvetković-Ilić and B. Zheng, "Weighted generalized inverses of partitioned matrices in Banachiewicz-Schur form," Journal of Applied Mathematics \& Computing, vol. 22, no. 3, pp. 175-184, 2006.

[22] J. Miao, "General expressions for the Moore-Penrose inverse of a $2 \times 2$ block matrix," Linear Algebra and its Applications, vol. 151, pp. 1-15, 1991.

[23] Y. Wei, "Expression for the Drazin inverse of a $2 \times 2$ block matrix," Linear and Multilinear Algebra, vol. 45, no. 2-3, pp. 131146, 1998.

[24] A. Ostrowski, "A new proof of Haynswortus quotient formula for Schur complement," Linear Algebra and its Applications, vol. 4, no. 4, pp. 389-392, 1971. 


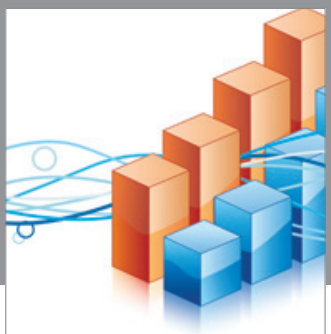

Advances in

Operations Research

vatem alat4

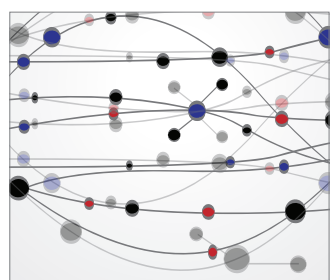

\section{The Scientific} World Journal
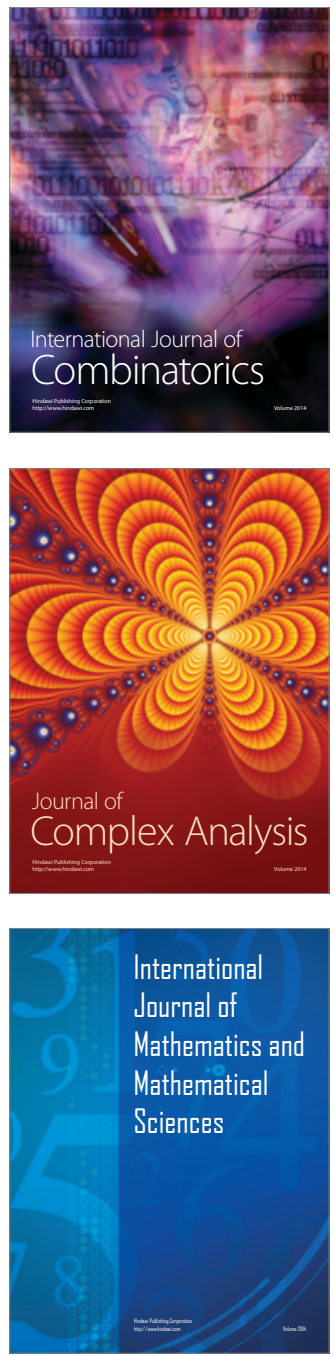
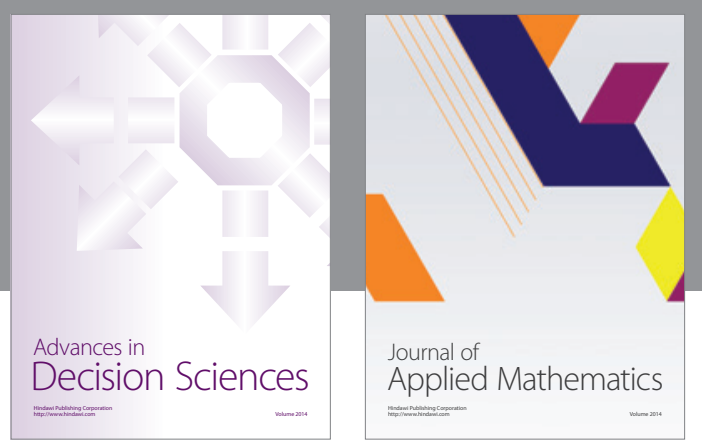

Algebra

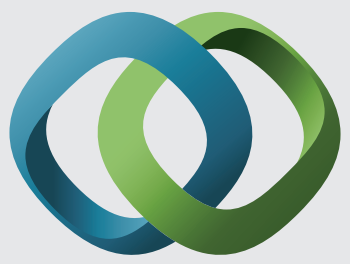

\section{Hindawi}

Submit your manuscripts at

http://www.hindawi.com
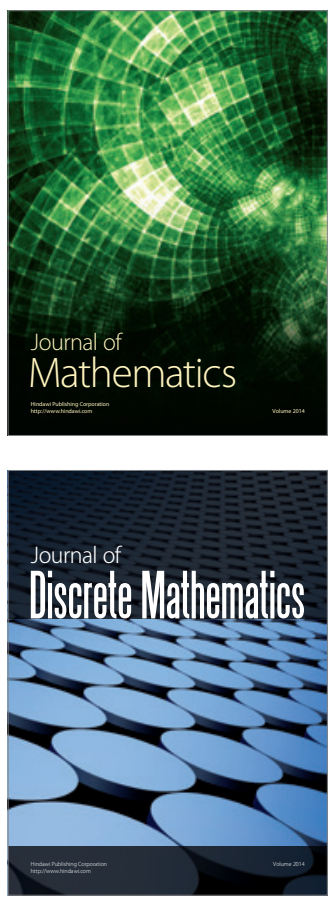



Mathematical Problems in Engineering
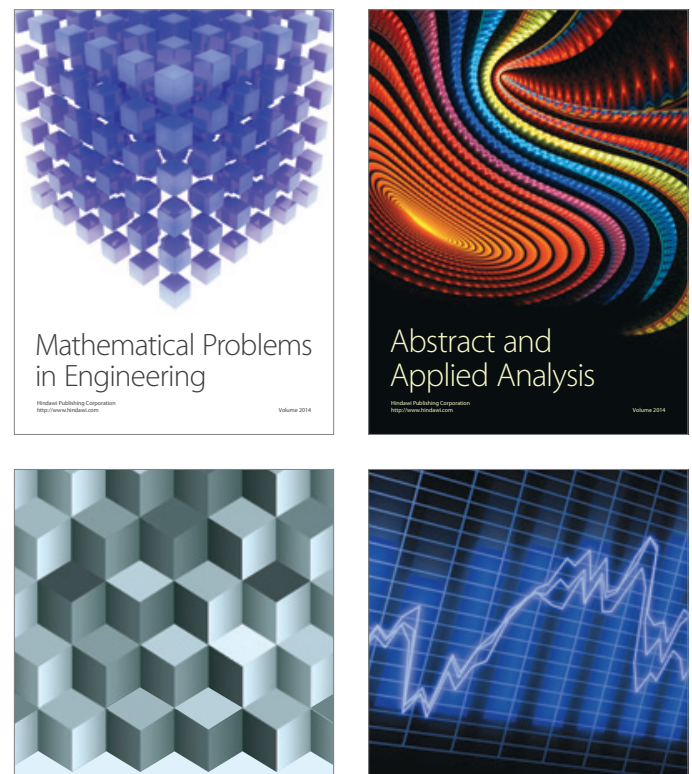

Journal of

Function Spaces

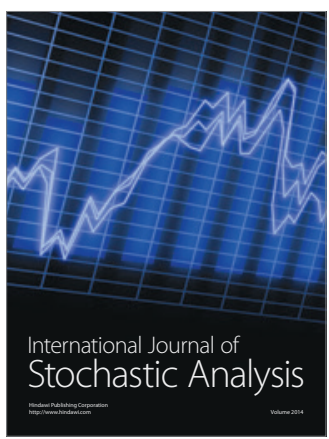

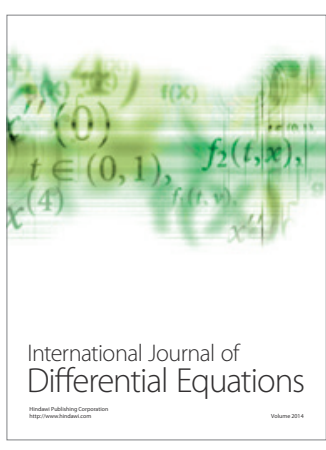
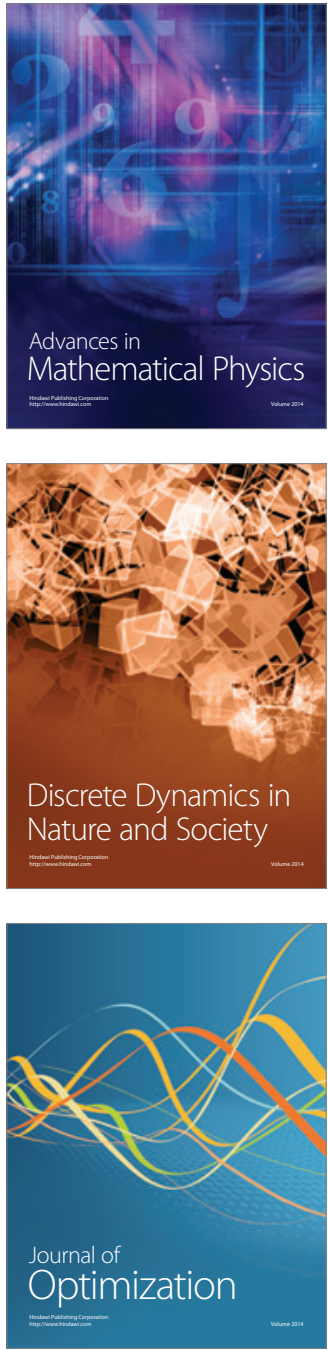